\title{
Virtual Fences
}

\author{
Kavyashree B S, Navarathna M, Samyak V Jain, Vignesh N, Prof. Vidyashree K P
}

\begin{abstract}
In modern world, human-animal conflict has caused hindrance for wildlife conservation and protection of human settlements. A search for effective protection systems have been proposed and re-proposed every passing day. Until now, wild animal identification and repelling systems have been created using Camera Surveillance, infrared and thermal sensors, LVDT, Geophones and Acoustic sensors. As these methods are very expensive and less accurate, an automated system for identification of human intruders and wild animals (Elephants and wild-boars) and a repelling alarm system is introduced in this project wherein the human-wildlife conflict can be reduced to a large extent. In this method, an amalgamation of three layers are deployed to identify the presence of animals. The first outer layer detects the movement with the help of a PIR (passive infrared detector) sensors and gives the warning signal to alert the people around that area using GSM as well as triggers the image detection part of the system. Middle layer activates the initial repelling system to make the animals realize that it is in a human habitat and to make them run back to the forest by triggering an alarm system of high frequency noise. In case if it is a human being trespassing into the farmland, a GSM model is used to send an alert text to the owner of the plot, informing him of the presence of an intruder. If the animals are still moving forward towards the conserved area, the third layer activates the second repelling system wherein, the alarm is produced through high-amplitude speakers along with high intensity search lights and fog dispensers. As the speakers and search lights are installed in patterns the entire attack susceptible area is covered, which reduces manual monitoring of the system. This system also holds good for identification and alerting when a human being trespasses the covered area, with intentions of causing theft or damage to the property.
\end{abstract}

Keywords : Alarm system, intruder detection, animal detection, repelling system, virtual fences.

\section{INTRODUCTION}

$\mathrm{T}_{\mathrm{t}}$ he population of wildlife is greatly intimidated by the changing human behaviour by means of vigorous natural resource accession and topographical changes. There has also been an increase in conflicts between human beings and wildlife. As a result of this a lot of resources have been compromised and need for compensation has increased. This

Revised Manuscript Received on December 15, 2019.

* Correspondence Author

Kavyashree B S*, Information science Engineering, Vidyavardhaka College of Engineering, Mysore, India. Email: bskavyashree1997@gmai.com

Navarathna M, Information science Engineering, Vidyavardhaka College of Engineering, Mysore, India.

Samyak V Jain, Information science Engineering, Vidyavardhaka College of Engineering, Mysore, India.

Vignesh N, Information science Engineering, Vidyavardhaka College of Engineering, Mysore, India.

Prof.Vidyashree K P, Information science Engineering, Vidyavardhaka College of Engineering, Mysore, India. Email: vidyashreekp@ vvce.ac.in has created a major hurdle for effective management of resource as well as wildlife conservation. In addition, theft of farm produce and livestock and farm equipment is at rife. Hence, a system to identify the culprits and to alert the owners of presence of intruders is essential. Be it wild animals or human intruders, the proposed system provides an apt solution. Property, human lives and wildlife conservation can be achieved by means of the proposed virtual fence system. Various methods have been adopted previously to ward off wild animals and intruders in Indian farmlands. Some of them were rifle-guns, and electric fences, beehives with live bees and scarecrows, out of which, many caused large scale destruction to human as well as animal lives. As technology improved, many bloodless solutions have been realized. Among them many were ineffective as they did not take physical, technological, climatic factors into consideration. Particularly, since elephants are large in size, it is difficult to protect ourselves against them. So a psychological understanding of their behaviour is important. As a simple solution to all these problems and as an amalgamation of systems to provide a single system to protect farmlands from wild animals as well as from intruders, we have come up with the concept of virtual fences.

\section{LITERATURE SURVEY}

Research on animal detection by human eyesight:

Many researches have been carried out to observe how quickly human eyes can detect the presence of a wild animal in a natural environment. From computational viewpoint, animal detection by human eyes is considered to be much reliable than other methods. In a research paper, it is cited that when a human being observes a picture involving an animal in a natural scene, he can detect the animal presence within $150 \mathrm{~ms}$ [7]. Though human eyes are reliable and efficient, a human being observing a scene can easily get tired. This reduces the efficiency and effectiveness. In addition, human eyes cannot only perform animal detection throughout the day. These limitations can be overcome by using computer technology in image processing for animal and human intruder detection.

Research on animal detection using image processing approach:

A research studying the locomotive behaviour of animals incorporated a method which was a combination of facial detection and tracking of target animals [8] using Haar-like feature, which are digital image features used for object detection along with ADA boost classifiers. To reduce junk images and to prolong battery life of the cameras, the video recorders are only turned on when the system is confident that the target animal has been spotted. Various local contrast configuration of luminescence channel to detect the image involving animals are used to detect animals using face detection systems. 


\section{Virtual Fences}

Along with these methods, a set of new developments have been reached in this field: According to a paper on Elephant intrusion detection, a system was developed which detects the elephant intrusion, creates an alert. But the drawback here was the use of GSM as alert option [1]. Any alert sent to mobile phones during an animal raid is not fruitful in real time situations. Hence, a more effective alarm system is required. Use of sensor networks in overseeing, tracking and determination of different wild organisms is a common practice that is carried out. For smaller animals and birds, RF tags are used to track their motion using radio telemetry [2]. This is a costly solution and these RF tags have to be replaced after their life span. To overcome these limitations a new technology is needed that enables new ways to monitor wildlife. A sensor- network-based tracking system is used for detection of bats by a research team. A system's working, its power saving techniques, information storage, and communication indication, information transfer and its low cost is beneficial in a real world bird-tracking system as proposed by a team of researchers [3]. To overcome limitation of previous approach, tracking of wildlife using radio collars was implemented. This approach also reduced implementation cost. Radio collar was tied around animal's neck which generated very high frequency. But, researchers had to physically go into the field and try to pin point the presence of animals using directional antenna. This approach was time consuming. To improve on this approach an aircraft drone with radio on-board was used which received the signal and collar position of the animal traced [4]. This again incurred a lot of expense. A system known as Wild CENSE was developed to study the migration of swamp deer using a network of wireless sensors. In this approach climatic and positional information of animal was collected and sent to base station using peer-to-peer network. Radio transceivers were used to transmit data to base station. From each peer node an external data flash memory was used to document the collected information. The collected information would be sent to database server via internet [5]. Similarly, a system is proposed in paper [6] which states that the system uses an RFID module and a GSM for alert purposes. But this is system also uses GSM for alert. In real time as a message won't help drive away crop raiding animals instantly. In 2005, JD wood and team proposed a system[9] which used seismic geophones to detect animal presence based on their footstep vibrations. The drawback of this system was, it did not consider the climatic impact. The moisture content on the ground did not allow sensors to detect vibrations. In 2017, D Karthik and team proposed a system[10]. Here, the system records images of the crop field $24 \times 7$ to detect any movement in the field. This was not a viable solution as junk image values slowed the system. In 2005, Navneet Dalal and team proposed a system[11], to detect human beings using HOG and SVM features. It used a concept of sliding window which was introduced to reduced false results and increased efficiency. It is otherwise very slow in detection as the sliding window takes time to process the image for object detection. In 2016, Mrs. Jalaja and team proposed a system[12], which uses PIR sensors and cameras to detect images. The drawback here is the use of MATLAB, which costs for license. Also debugging as well as converting a code from MATLAB can be a difficult process. In 2016, Sachin Umesh Sharma and team proposed a system[13], which prevents animal collision with vehicles. It used a set of cameras which are mounted on the vehicle, which will detect animals nearby and alerts the driver.

This system doesn't work as the reaction time of both the system and the driver can be slower.

PIR sensor: Is passive, hence doesn't vent infrared rays but only receives radiation emitted or reflected from objects in its range.

GSM: A cellular technology used to transmit digital and mobile messages.

Raspberry pi: A SIM card sized, capable computer device used to connect multiple systems.

Tensor flow: An end-to-end open source machine learning platform. Used for artificial neural networking and other machine learning applications.

\section{RESUTLS AND DISCUSSIONS}

Objective: This system is particular to farmlands adjacent to the forest areas, but is scalable. The system is also particular to detection of only human beings, elephants and wild-boars which is also scalable.

The proposed system takes various factors into account. It can be used for detection and repelling of wild animals as well as human intruders. The system consists of 3 layers. The first layer includes a PIR module which detects the presence of an organism and an alert text message is sent to the owner of the plot. Also, a trigger is sent to capture image to detect the presence of the animal or human intruder here on referred to as the 'pest'. The image capture is triggered only after the PIR module detects variations to reduce junk image values. The cameras are mounted on towers or trees at a height of 10 feet. The image processing unit includes a set of pre-processing activities such as:

Mask operation: An image processing operation used to make an object stand out by isolating it from its background. This can also be used to place that object on a different background.

Image differencing: This is one of the image processing techniques used to notice and record differences between images. A resultant image is produced based on the difference between each pixel of each of those two images. This technique works only when complementary points of both the images coincide and their values must be accordant, either by careful configuration or by post-processing techniques. The intricacy of the image pre-processing technique which is needed before image differencing alters with the type of image being processed.

Multiple Thresholding: This is a simple image processing technique used in image segmentation. A binary image can be created from a grayscale or full- colour image. This is basically done to separate the object or pixels in foreground from background pixels to enhance image view.

Filtering: This is also an image processing technique where some features in an image can be enhanced. Similarly unwanted features can also be deleted from a picture if it is not necessary. 
The figure 1 shows the work flow of the virtual fences. The process that occurs from the detection of intruders by a PIR system to each intruder specific actions that are taken is mentioned. Figure 2 shows the Image processing action diagram. It tells us how the system detects an intruder and classifies them based on their key features using image processing techniques.Figure 3 represents the structure and architecture of the system. The pacing of the system components for a given plot is observed,

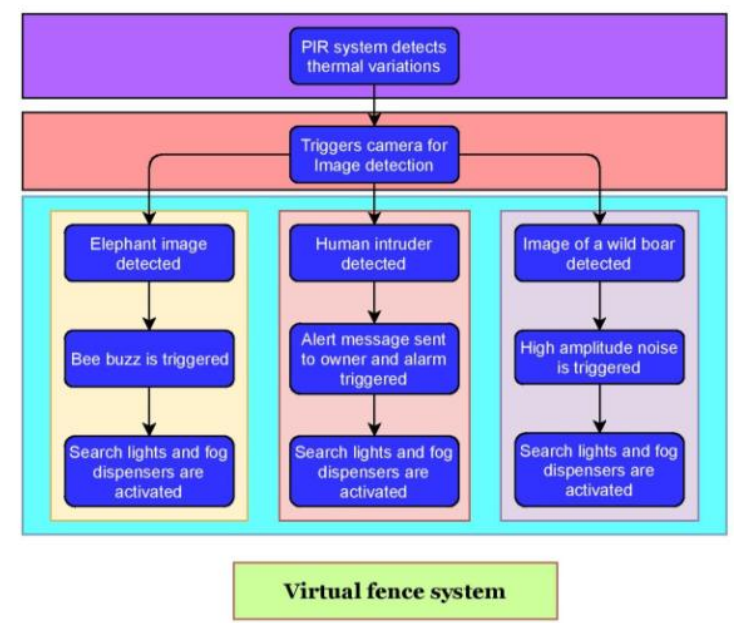

Fig 1: Virtual fences workflow diagram

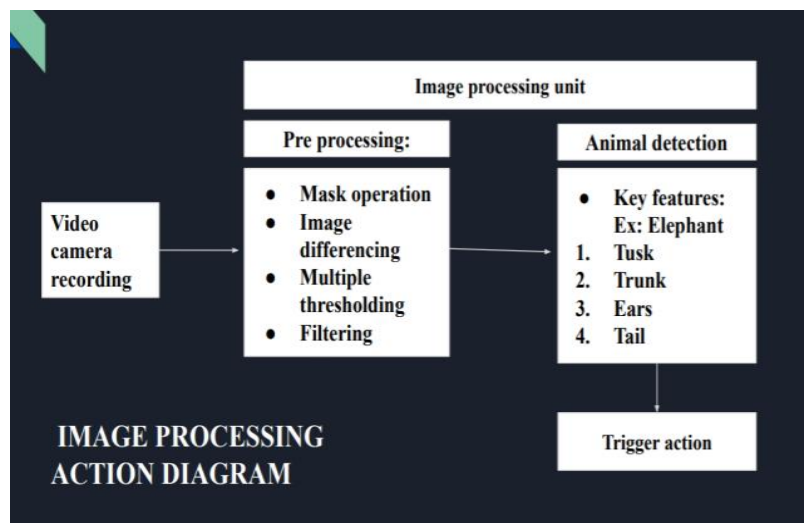

Fig 2: Image processing action diagram

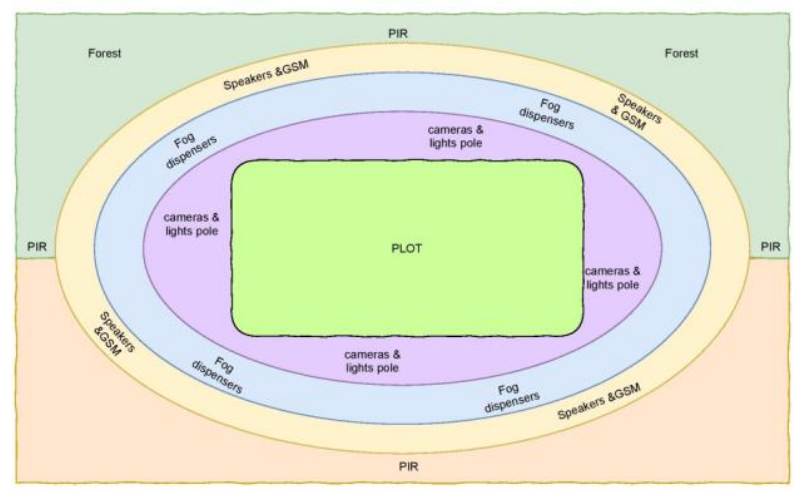

Fig 3: Architecture of the system

After all the pre-processing techniques have been realized, the pest is detected by matching the predefined key features. These key features are compared with images stored using the Tensor flow source. The key features for every organism differs from the other. As an example, the key features for an elephant are: Tusk, trunk, ears and tail. These features are particular to elephants alone. This selection of key features includes the front, peripheral as well as the appearance of an elephant from behind. Similarly, the key features differ from organism to organism. After initial detection, if the image detected is of a human being, the person is considered as an intruder and an alert text message is sent to the plot owner through the GSM module and a buzzer alarm is also triggered. If the image detected is of an elephant, the sound of bee buzz is initiated via a set of strategically placed high-amplitude speakers. As elephants are huge it size, it is difficult to tackle them by us human beings. Thus, we must gain the upper hand by using psychological methods. A study conducted by Oxford researcher Lucy King observed that Elephants fled on hearing bee buzz. This concept has been implemented using installation of live beehives throughout the world. But the bee sting can prove fatal to both elephant calves and human beings. Not to forget, the maintenance of beehives is very difficult. If the image detected is not any of these, a normal high-amplitude alarm is turned on. This concludes the components of the second layer. The third layer includes a series of search lights as well as fog dispensers. The search lights are installed on towers which are at a height of 8 feet. The fog dispensers are at the ground level as they mostly target smaller but ferocious animals like wild boars. All these layers are at a distance of 15 meters from the actual farmlands.

\section{CONCLUSION AND FUTURE WORK}

As a conclusion of these researches, we have come up with a system called "Virtual fences" which makes use of psychological, physical and technological methods to detect animal and intruder presence and provides an efficient alarm and repel system. The idea of virtual fences is scalable. It can be implemented places other than farmlands, taking necessary constraints into consideration.

\section{REFERENCES}

1. N. Rajathi, Farithul Inzamam M, N. Suganthi, Elephant Intrusion Detection and Repulsive System, ISSN: 2277-3878, Volume-7 Issue-4S, November 2018

2. Tao Yang, Xiaohan Liu, Baoping Yan "Research on the Architecture of Wildlife Observation and Communication System" Computer Network Information Center (CNIC) Chinese Academy of Sciences Beijing, China, IEEE, 2015

3. Mehmet C. Vuran, William P. Bennett, Jr., Matthew B. Dwyer, Anne Lacy, Sebastian Elbaum, Mike Engels, Walter Wehtje "Sensing Through the Continent: Towards Monitoring Migratory Birds Using Cellular Sensor Networks", IEEE April 2012.

4. Aman Kumar, Ravi Bagree, PrabhatRanjan, Vishwas Raj Jain, “ wildCENSE : GPS based Animal Tracking System", IEEE, 2008.

5. Thorsten Nowak, Christopher Eibel, BjörnCassens, Frieder Mayer, Klaus Meyer-Wegener ,Simon Ripperger, Martin Hierold, and Alexander Kölpin, Falko Dressler, "From Radio Telemetry to Ultra-Low-Power Sensor Networks: Tracking Bats in the Wild IEEE communication magazine, January 2016 .

6. Y. Dhamodharan, C S. Santhosh, N E. Kavi Priya, S. Santhiya M.Surekha, "A SMART FARMLAND USING RASPBERRY PI CROP PREVENTION AND ANIMAL INTRUSION DETECTION SYSTEM", e-ISSN: 2395-0056 p-ISSN: 2395-0072

7. A. Delorme, and S. T. C. Marlot, M. F. Thorpe, "A limit to the speed processing in ultra-rapid visual categorization of novel natural scene," Cognitive Neuroscience, pp. 171-180, 2003 


\section{Virtual Fences}

8. T. Burghardt and J. Calic, "Real-time face detection and tracking of animals," 8th Seminar on Neural Network Applications in Electrical Engineering, pp. 27-32, 2006.

9. Caitlin O'Connell-Rodwell, J D Wood, S L Klemperer, Using seismic sensors to detect elephants and large mammals, Journal of applied ecology, 2005

10. R. Ramesh Babu, Dugyala Karthik, " Smart Crop Protection System with Image Capture over IOT”, IJAIST, November 2017

11. Bill Triggs \& Navneet Dalal, Histograms of Oriented Gradients for Human Detection, IEEE, 2005

12. Mrs. S. Jalaja, Abel Daniel T, Gowtham Shankar B, Kaliraj M, Yuthish Khanna J, Real Time Animal Repellent System using Image Processing, IJSRD, 2016

13. Dharmesh J Shah and Sachin Umesh Sharma, A Practical Animal Detection and Collision Avoidance System Using Computer Vision Technique, IEEE, 2016

\section{AUTHORS PROFILE}

VIDYASHREE $\mathbf{K} \mathbf{P}$ is a graduate from V.V.I.E.T college Mysore under VTU, Belgaum and did her M. Tech in Computer Network Engineering from N.I.E, Mysore in the year 2013. She Worked as Intern @ LG Soft India PVT LTD, Bangalore for 1 year. She served as Lecturer in the Dept. of Computer Science and Engg. at P.E.S.C.E, Mandya for 6 months before joining VVCE. Currently serving as Assistant Professor in Information Science \& Engineering department. Her areas of interest are Computer Networks and Operating System. 